\title{
A New Type of Interval-valued Fuzzy Filters of Ordered Semigroups
}

\author{
Hidayat Ullah Khan ${ }^{1}$, Asghar Khan ${ }^{2}$, Faiz Muhammad Khan ${ }^{3}$ and Yongin $\mathrm{Li}^{4}$,* \\ ${ }^{1}$ Department of Mathematics, University of Malakand, Dir (L), Khyber Pakhtunkhwa, Pakistan \\ ${ }^{2}$ Department of Mathematics, Abdul Wali Khan University Mardan, Mardan, Khyber Pakhtunkhwa, Pakistan \\ ${ }^{3}$ Department of Mathematics and Statistics, University of Swat, Khyber Pakhtunkhwa, Pakistan \\ ${ }^{4}$ Department of Mathematics, Sun Yat-sen University, Guangzhou, 510275, P. R. China \\ ${ }^{*}$ Corresponding author
}

\begin{abstract}
One of the advantageous generalizations of Zadeh's fuzzy set theory is the concept of interval-valued fuzzy subset. This generalization of fuzzy subset is frequently and successfully applied by engineers and scientists in the fields of Robotics, Control Theory and Computer Engineering. The purpose of this study is to investigate another generalization of $\mathbf{I}-\mathrm{V}\left(\in, \in \vee q_{\widetilde{k}}\right)$ -
\end{abstract}

fuzzy filters (in short I-V $\left(\in, \in \vee q_{\tilde{k}}\right)$-FFs). Therefore we employed the existing concept of I-V-FS theory in the structure of ordered semigroup to introduce I-V $\left(\bar{\epsilon}_{\tilde{\gamma}}, \bar{\epsilon}_{\tilde{\gamma}} \vee \bar{q}_{\widetilde{\delta}}\right)-$ FFs. In this research, investigating the relationship of ordinary intervalvalued fuzzy filters (in short I-V-FFs) with these new type I-V-FFs, a condition is determined under which I-V-FFs of type $\left(\bar{\epsilon}_{\tilde{\gamma}}, \bar{\epsilon}_{\tilde{\gamma}} \vee \bar{q}_{\tilde{\delta}}\right)$ will be ordinary I-V-FFs. Finally, ordinary I-V-FFs are linked with these new notions by means of I-V level subsets.

Keywords-filters; ordered semigroups; fuzzy filters; intervalvalued fuzzy subset; interval-valued fuzzy left (right) filters; interval$\operatorname{valued}\left(\bar{\epsilon}_{\tilde{\gamma}}, \bar{\epsilon}_{\bar{\gamma}} \vee \bar{q}_{\widetilde{\delta}}\right)$-fuzzy filters

\section{INTRODUCTION}

First of all, we give a list of abbreviations (see Table I) that are used frequently in this paper.

TABLE I. ABBREVIATIONS

\begin{tabular}{|l|l|}
\hline \multicolumn{1}{|c|}{ Name } & \multicolumn{1}{c|}{ Abbreviation } \\
\hline Interval-valued Fuzzy Subset & I-V-FS \\
\hline Interval-valued Fuzzy Filters & I-V-FFs \\
\hline Interval-valued Fuzzy Left Filter & I-V-FLF \\
\hline Interval-valued Fuzzy Right Filter & I-V-FRF \\
\hline Fuzzy Left Filter & FLF \\
\hline Fuzzy Right Filter & FRF \\
\hline Inter-valued $\left(\bar{\epsilon}_{\tilde{\gamma}}, \bar{\epsilon}_{\tilde{\gamma}} \vee \bar{q}_{\tilde{\delta}}\right)$-fuzzy filters & I-V $\left(\bar{\epsilon}_{\tilde{\gamma}}, \bar{\epsilon}_{\tilde{\gamma}} \vee \bar{q}_{\tilde{\delta}}\right)-$ \\
\hline interval-valued $\left(\in, \in \vee q_{\tilde{k}}\right)$-fuzzy filters & FFs \\
\hline Interval-valued fuzzy point $\left(\in, \in \vee q_{\tilde{k}}\right)-F F s$ \\
\hline A & I-V-FP \\
\hline
\end{tabular}

According to Gorzałczany, "The fundamental reason of introducing the concept of I-V-FS (extension of fuzzy set) is associated with the fact that the membership functions of fuzzy set representing particular verbal expressions cannot be defined unmistakably on the basis of available information. Therefore, it is not always possible for membership functions of type $f: Y \rightarrow[0,1]$ to associate with each point " $x$ " in a set $Y$ a single point in the unit closed interval without the loss of some valuable information" [1]. In fuzzy decision-making problems, the memberships' grades are very individual and usually depends on the assessment of individual experts and thus cannot be confirmed. For example, suppose there are five individual experts giving their evaluations regarding someone as $0.30,0.45$, $0.55,0.60$ and 0.80 , respectively. Clearly, the evaluation of an individual's capability by an interval $[0.30,0.80]$ is more practical and authentic than a single-point value. There, we observe that I-V-FS theory is one of the useful generalization of fuzzy set, independently presented by Zadeh [2], GrattanGuiness [3] and Jahn [4] at the same time. Extending the idea of Rosenfeld's fuzzy subgroups [5], Biswas [6] for the first time employed the concept given in [2-4] in groups and coined the idea of I-V fuzzy subgroup. Davvaz [7] further extended the concept of [6] to hyper structure and initiated the idea of I-V fuzzy subhypergroup. Let $S \neq \phi$ and "。" be an associative binary operation on $S$, then $(S, \circ)$ is knows as a semigroup. Narayanan and Manikantan [8] employed I-V-FS theory in semigroup and initiated the concepts of I-V fuzzy ideals. They further characterized semigroups in terms these I-V fuzzy ideals. If $(S, \circ)$ is a semigroup, $(S, \leq)$ is a po-set and $a \leq b$ implies $a \cdot x \leq b \cdot x$ and $x \cdot a \leq x \cdot b$ for each $x, a, b \in S$, where $\leq$ is an ordered relation, then $(S, \circ, \leq)$ is called an ordered semigroup. Extending the concept of [8] to ordered semigroups Shabir and Khan [9] and defined the concepts of I-V fuzzy ideals. Consider a function $f_{A}: S \rightarrow[0,1]$, then the set $A=\left\{\left(y, f_{A}(y)\right)\right.$ for $\left.y \in S\right\}$ representing a fuzzy subset of $S$, where $f_{A}: S \rightarrow[0,1]$ is called membership function. If $f_{A}$ takes a non-zero value only at a single point $y \in S$ (say), that is, $f_{A}(y)=\theta \in(0,1]$ and $f_{A}(z)=0$ for all $y \neq z$ in $S$, then $A$ is called a fuzzy point and is denoted by $y_{\theta}$, where $y$ is the support and $\theta$ is the value of the fuzzy point $y_{\theta}$. The relations of a fuzzy point and a fuzzy subset was studied by Pao-Ming, Ying-Ming [10] and Murali [11]. A fuzzy point $y_{\theta}$ belongs to a fuzzy subset if and only if $f_{A}(y) \geq \theta$ and therefore we can say 
that a fuzzy set can be written as the union of all fuzzy points contained in $A$. On the other hand if $f_{A}(y)+\theta>1$, then $y_{\theta}$ is said to quasi-coincident with $A$. These relations of a fuzzy point and a fuzzy set played an important role to provide more generalized form of Rosenfeld's fuzzy subgroups. Using theses relations between a fuzzy points and fuzzy subsets, Bhakat and Das [12] extended the idea of Rosenfeld's fuzzy subgroups and indtroduced the concept of $(\in, \in \vee q)$-fuzzy subgroups. By using the combined notions of fuzzy point belongingness and quasicoincident relations with a fuzzy subset Davvaz and Khan [13] generalized the ideas of Kehayopulu and Tsingelis [14] and defined fuzzy filters of type $(\in, \in \vee q)$ and $(\bar{\epsilon}, \bar{\in} \vee \bar{q})$. Jun et al. [15] further study the ideas of [14] and defined the notion of $\left(\in, \in \vee q_{k}\right)$-fuzzy left (right) filters of ordered semigroups. In addition, Davvaz et al. [16] and Khan et al. [17] further generalized the concepts given in $[14,15]$ and defined respectively the concepts of $\mathrm{I}-\mathrm{V} \quad\left(\in, \in \vee q_{\widetilde{k}}\right)-\mathrm{FFs}$ and I-V $\left(\in_{\tilde{\gamma}}, \in_{\tilde{\gamma}} \vee q_{\widetilde{\delta}}\right)$-FFs of ordered semigroup.

In this paper, we further study the concepts given in $[16,17]$ and try to obtain another generalization of the concepts defined in [13-17]. In this regard the notion of I-V $\left(\bar{\epsilon}_{\tilde{\gamma}}, \bar{\epsilon} \tilde{\gamma} \vee \bar{q} \widetilde{\delta}\right)$-fuzzy filters is defined and some properties of ordered semigroups is investigated by the properties this new type of I-V-FFs. A condition under which an interval-valued fuzzy filter of type $\left(\bar{\epsilon}_{\gamma}, \bar{\epsilon}_{\gamma} \vee \overline{\mathrm{q}}_{\tilde{\delta}}\right)$ will be an interval-valued fuzzy filter is determined. Finally, interval-valued fuzzy filters are linked with these new notions by means of interval-valued level subsets.

\section{PRELIMINARIES}

We need to recall some fundamental concepts that are required to carry out this study easily. Throughout this paper an ordered semigroup is represented by $S$ unless otherwise stated.

For a non-empty subsets $A$ and $B$ of $S$ we have the sets

$$
(A]:=\{x \in S \text { such that } x \leq y \text { for some } y \in A\},
$$$$
A B:=\{a b \text { such that } a \in A, b \in B\} .
$$

Let $T \subseteq S$ such that $T \neq \phi$ and $T T \subseteq T$. Then the set $T$ is called a subsemigroup of $S$.

Let $\mathrm{Z} \subseteq S$ such that $\mathrm{Z} \neq \phi$. Then $\mathrm{Z}$ is called a left filter of $S$ whenever the following statements hold simultaneously:

(b) $a \in \mathrm{Z}$ implies $b \in \mathrm{Z}$ for all $a \leq b$,

$\left(b_{2}\right) a \in Z$ and $b \in Z$ implies $a b \in Z$,

(b) $a b \in \mathrm{Z}$ implies $a \in \mathrm{Z}$.

If $\mathrm{Z}$ satisfy Statements $\left(b_{1}\right),\left(b_{2}\right)$ and the following statement simultaneously, then $Z$ is called a right filter:

$\left(b_{4}\right) a b \in \mathrm{Z}$ implies $b \in \mathrm{Z}$.

If $\mathrm{Z}$ is a left as well as right filter, then $\mathrm{Z}$ is said to be a two sided filter or simply a filter.
Next we give some fundamental fuzzy concepts below:

The set $\mathbf{F}=:\left\{\left(y, \mu_{\mathbf{F}}(y)\right)\right.$ such that $\left.y \in S\right\}$ represents a fuzzy subset of $S$, whereas $\mu_{\mathbf{F}}: S \rightarrow[0,1]$ is a membership function.

If the following statements hold simultaneously for all $x, y \in S$, then $\mathbf{F}$ is called FLF of $S$ :

$\left(b_{5}\right) \quad y \geq x$ implies $\mu_{\mathbf{F}}(y) \geq \mu_{\mathbf{F}}(x)$.

$\left(b_{6}\right) \min \left\{\mu_{\mathbf{F}}(x), \mu_{\mathbf{F}}(y)\right\} \leq \mu_{\mathbf{F}}(x y)$,

$\left(b_{7}\right) \quad \mu_{\mathbf{F}}(x y) \leq \mu_{\mathbf{F}}(x)$.

If the Statements $\left(b_{5}\right),\left(b_{6}\right)$ given above and the Statement $\left(b_{8}\right)$ given below hold simultaneously, then $\mathbf{F}$ is FRF of $S$ :

(bs) $\mu_{\mathbf{F}}(x y) \leq \mu_{\mathbf{F}}(y)$.

If $\left(b_{5}\right),\left(b_{6}\right),\left(b_{7}\right)$ and $\left(b_{8}\right)$ hold simultaneously, then $\mathbf{F}$ is a fuzzy filter.

Now we recall some interval-valued fuzzy concepts.

In this study, for $0 \leq a^{-} \leq a^{+} \leq 1$ we denote $\left[a^{-}, a^{+}\right]$(closed interval) by $\tilde{a}$ and $[a, a]$ (point interval) is simply represented by $a$ in $[0,1]$ (unit closed interval). Take $j$ from $\Omega$ (indexing set) and the closed sub-intervals $\tilde{a}_{j}=\left[a_{j}^{-}, a_{j}^{+}\right], \widetilde{b}_{j}=\left[b_{j}^{-}, b_{j}^{+}\right]$ from $D[0,1]$, where $D[0,1]$ denotes the set of all closed sub intervals of the unit closed interval. Then the following operations are defined:

- $\quad \tilde{a}_{i} \leq \tilde{b}_{i}$ if and only if $a_{i}^{-} \leq b_{i}^{-}$and $a_{i}^{+} \leq b_{i}^{+}$,

- $\tilde{a}_{i}=\tilde{b}_{i}$ if and only if $a_{i}^{-}=b_{i}^{-}$and $a_{i}^{+}=b_{i}^{+}$,

- $\tilde{a}_{i}<\tilde{b}_{i}$ if and only if $\tilde{a}_{i} \leq \tilde{b}_{i}$ and $a_{i}^{-} \neq b_{i}^{-}, a_{i}^{+} \neq b_{i}^{+}$,

- $\tilde{a}_{i}+\tilde{b}_{i}=\left[a_{i}^{-}+b_{i}^{-}, a_{i}^{+}+b_{i}^{+}\right]$,

- $\tilde{a}_{i}+\tilde{b}_{i}=\left\{\begin{array}{lll}{\left[a_{i}^{-}-b_{i}^{-}, a_{i}^{+}-b_{i}^{+}\right],} & \text {if } a_{i}^{-}+b_{i}^{-}<a_{i}^{+}+b_{i}^{+}, \\ {\left[a_{i}^{+}+b_{i}^{+}, a_{i}^{-}+b_{i}^{-}\right],} & \text {if } \quad a_{i}^{-}+b_{i}^{-} \geq a_{i}^{+}+b_{i}\end{array}\right.$,

For $0 \leq k \leq 1, k \widetilde{a}_{i}=\left[k a_{i}^{-}, k a_{i}^{+}\right]$.

An I-V-FS $I$ defined on a $S$ is denoted and defined as $I=:\left\{\left(y, \widetilde{f}_{I}(y)=\left[f_{I}^{-}(y), f_{I}^{+}(y)\right]\right)\right.$ such that $\left.y \in S\right\} \quad$, where $f_{I}^{-}(y), f_{I}^{+}(y)$ are fuzzy with $0 \leq f_{I}^{-}(y) \leq f_{I}^{+}(y) \leq 1$ for all $y \in S$. The interval $\widetilde{f}_{I}(x)=\left[f_{I}^{-}(x), f_{I}^{+}(x)\right]$ denotes the degree of the membership of an element $y$ in $I$. If $f_{I}^{-}(x)=f_{I}^{+}(x)=a$ (say), then $0 \leq a \leq 1$ and therefore $\tilde{f}_{I}(x)=[a, a]$ and from this point see a fuzzy subset as a special case of I-V-FS. For the sake of convenience it is assumed that $[a, a] \in D[0,1]$ and hence $\widetilde{f}_{I}(x) \in D[0,1]$ for all $x \in X$. From the above discussion it is clear that an I-V-FS $I$ is characterized 
respectively by the lower and upper limits $f_{I}^{-}(y), f_{I}^{+}(y)$ of $\tilde{f}_{I}(x)$.

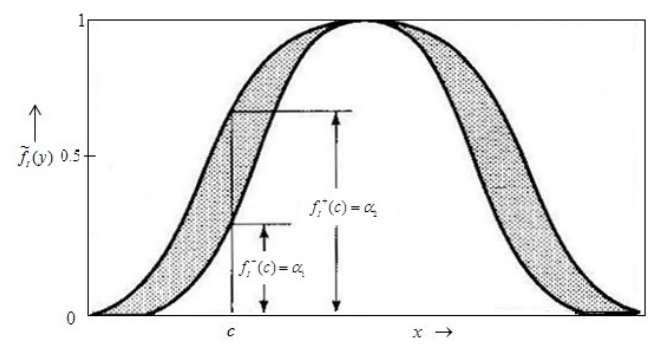

FIGURE I. I-V-FS $\tilde{f}_{I}(c)=\left[\alpha_{1}, \alpha_{2}\right]$

For the I-V membership function $\tilde{f}_{I}(y)=\left[f_{I}^{-}(y), f_{I}^{+}(y)\right]$ and $[0,0] \leq \widetilde{\theta}=\left[\theta^{-}, \theta^{+}\right]<[1,1]$ we denote and define $\widetilde{f}_{I}(y)+\widetilde{\theta}=\left[f_{I}^{-}(y)+\theta^{-}, f_{I}^{+}(y)+\theta^{+}\right]$for all $y \in S$. Whenever $f_{I}^{-}(y)+\theta^{-}>1$ and $f_{I}^{+}(y)+\theta^{+}>1$, then we write $\tilde{f}_{I}(y)+\widetilde{\theta}>[1,1]$.

An I-V-FS $I$ of an ordered semigroup $S$ of the form

$$
\tilde{f}_{I}(z):=\left\{\begin{array}{ccc}
\widetilde{\theta} \neq[0,0], & \text { if } & z=y \\
{[0,0],} & \text { if } & z \neq y
\end{array}\right.
$$

is called an I-V-FP with value $\tilde{\theta}$ and support $y$ and is represented by $y_{\tilde{\theta}}$. The I-V-FP $y_{\tilde{\theta}}$ is said to be contained or belongs to the I-V-FS $I$ if $\tilde{f}_{I}(y) \geq \widetilde{\theta}$ and is denoted by $y_{\tilde{\theta}} \in I$. On the other hand, if $\tilde{f}_{I}(y)+\widetilde{\theta}>[1,1]$ then the I-V-FP $y_{\tilde{\theta}}$ is said to be quasi-coincident with $I$ and is written as $y_{\tilde{\theta}} q I$. We write $y_{\tilde{\theta}} \in \vee q I$ if $y_{\tilde{\theta}} \in I$ or $y_{\tilde{\theta}} q I$.

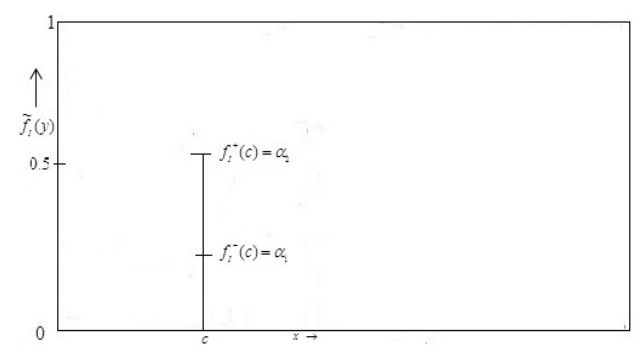

FIGURE II. I-V-FP $\widetilde{f}_{I}(c)=\left[\alpha_{1}, \alpha_{2}\right]$ AND $\widetilde{f}_{I}(x \neq c)=[0,0]$

For each $\tilde{\theta}$ taken from $D[0,1]$ the crisp set $U(I ; \widetilde{\theta})=:\left\{y \in S\right.$ such that $\left.\widetilde{f}_{I}(y) \geq \widetilde{\theta}\right\}$ is called level set of $I$.

The I-V-FS $I$ of $S$ is known as I-V-FLF if the following statements hold simultaneously for each $x, y$ in $S$ :

$\left(b_{9}\right) \quad y \geq x$ implies $\tilde{f}_{I}(y) \geq \tilde{f}_{I}(x)$, $\left(b_{10}\right) \widetilde{f}_{I}(x y) \geq \mathrm{r} \min \left\{\tilde{f}_{I}(x), \tilde{f}_{I}(y)\right\}$,

(b $\left.b_{11}\right) \tilde{f}_{I}(x) \geq \widetilde{f}_{I}(x y)$.

If Statements $\left(b_{9}\right),\left(b_{10}\right)$ and Statement $\left(b_{12}\right)$ (given below) hold simultaneously for each $x, y$ in $S$, then $I$ is called an IV-FRF fuzzy right filter:

$$
\left(b_{12}\right) \tilde{f}_{I}(x y) \leq \tilde{f}_{I}(y) .
$$

If $I$ is both I-V-FLF and I-V-FRF, then $I$ is called I-V-FF.

\section{A NEW GENERALIZATION OF INTERVAL-VALUED FuZZY FILTERS}

Here, we provide a generalization of the concepts given in [13-17]. We introduce the notion of I-V $\left(\bar{\epsilon}_{\tilde{\gamma}}, \bar{\epsilon}_{\tilde{\gamma}} \vee \bar{q}_{\tilde{\delta}}\right)-$ FFs and discuss some characterizations of ordered semigroup.

Let denote by $D^{*}[0,1]$ a totally ordered subset of $D[0,1]$ and by $S$ an ordered semigroup and let $\widetilde{f}_{I}: S \rightarrow D^{*}[0,1]$.

Throughout this paper, we let $\tilde{\gamma}, \widetilde{\delta} \in D^{*}[0,1]$ such that $\left[\gamma^{-}, \gamma^{+}\right]=\tilde{\gamma}<\widetilde{\delta}=\left[\delta^{-}, \delta^{+}\right]$.

If $\tilde{f}_{I}(x) \geq \tilde{\theta}>\tilde{\gamma}$, then we say that the I-V-FP $y_{\tilde{\theta}} \tilde{\gamma}$ belongs to $I$ and is denoted by $x_{\tilde{t}} \in_{\tilde{\gamma}} I$. On the other hand, if $\widetilde{f}_{I}(x)+\widetilde{\theta}>\widetilde{\delta}+\widetilde{\delta}$, then we say that $y_{\widetilde{\theta}}$ is $\widetilde{\delta}$-quasi-coincident with $I$ and is denoted by $y_{\tilde{\theta}} q_{\tilde{\delta}} I$. If $x_{\tilde{t}} \in \tilde{\gamma}$ or $y_{\tilde{\theta}} q_{\tilde{\delta}} I$, then we write $y_{\tilde{\theta}} \in \in_{\tilde{\gamma}} \vee q_{\tilde{\delta}} I$. If $x_{\tilde{t}} \in_{\tilde{\gamma}} I$ and $y_{\tilde{\theta}} q_{\tilde{\delta}} I$, then we write $y_{\tilde{\theta}} \in_{\tilde{\gamma}} \wedge q_{\tilde{\delta}} I$. We say $y_{\tilde{\theta}} \bar{\alpha} I$, if, the relation $y_{\tilde{\theta}} \alpha I$ does not satisfy, where $\alpha$ represents an element from the set $\left\{\in_{\tilde{\gamma}}, q_{\tilde{\delta}}, \in_{\tilde{\gamma}} \vee q_{\tilde{\delta}}, \in_{\tilde{\gamma}} \wedge q_{\tilde{\delta}}\right\}$.

\section{A. Definition}

Let $I$ be an I-V-FS of $S$. If $I$ satisfy the following conditions simultaneously for all $x, y$ in $S$ and $\tilde{\gamma}<\tilde{\theta} \leq[1,1]$, then $I$ is called an $\mathrm{I}-\mathrm{V}\left(\bar{\epsilon}_{\tilde{\gamma}}, \bar{\epsilon}_{\tilde{\gamma}} \vee \bar{q}_{\tilde{\delta}}\right)-\mathrm{FLF}$ :

$$
\begin{aligned}
& \left(d_{1}\right) x \leq y \text { and } y_{\tilde{\theta}} \bar{\epsilon}_{\tilde{\gamma}} I \text { implies } x_{\tilde{\theta}} \bar{\epsilon}_{\tilde{\gamma}} \vee \bar{q}_{\tilde{\delta}} I, \\
& \left(d_{2}\right)(x y)_{\tilde{\theta}} \bar{\epsilon}_{\tilde{\gamma}} I \text { implies } x_{\tilde{\theta}} \bar{\epsilon}_{\tilde{\gamma}} \vee \bar{q}_{\tilde{\delta}} I \text { or } y_{\tilde{\theta}} \bar{\epsilon}_{\tilde{\gamma}} \vee \bar{q}_{\tilde{\delta}} I, \\
& \left(d_{3}\right) x_{\tilde{\theta}} \bar{\epsilon}_{\tilde{\gamma}} I \text { implies }(x y)_{\tilde{\theta}} \bar{\epsilon}_{\tilde{\gamma}} \vee \bar{q}_{\tilde{\delta}} I .
\end{aligned}
$$

If $I$ satisfy Statements $\left(d_{1}\right),\left(d_{2}\right)$ and Statement $\left(d_{4}\right)$ (given below):

$\left(d_{4}\right) y_{\tilde{\theta}} \bar{\epsilon}_{\tilde{\gamma}} I$ implies $(x y)_{\tilde{\theta}} \bar{\epsilon}_{\tilde{\gamma}} \vee \bar{q}_{\tilde{\delta}} I$ for each $x, y$ in $S$ and $\tilde{\gamma}<\tilde{\theta} \leq[1,1]$.

Then $I$ is called I-V $\left(\bar{\epsilon}_{\tilde{\gamma}}, \bar{\epsilon}_{\tilde{\gamma}} \vee \bar{q}_{\tilde{\delta}}\right)-\mathrm{FRF}$.

An I-V-FS $I$ is called an I-V $\left(\bar{\epsilon}_{\tilde{\gamma}}, \bar{\epsilon}_{\tilde{\gamma}} \vee \bar{q}_{\tilde{\delta}}\right)$-FF if it is I-V $\left(\bar{\epsilon}_{\tilde{\gamma}}, \bar{\epsilon}_{\tilde{\gamma}} \vee \bar{q}_{\tilde{\delta}}\right)-$ FLF as well as I-V $\left(\bar{\epsilon}_{\tilde{\gamma}}, \bar{\epsilon}_{\tilde{\gamma}} \vee \bar{q}_{\tilde{\delta}}\right)-$ FRF. 


\section{B. Example}

Define the order relation as:

$$
\leq=:\{(a, a),(a, d),(b, b),(c, c),(d, d)\},
$$

and multiplication on the elements of $S=\{a, b, c, d\}$ as given in Table II:

\section{TABLE II. MULTIPLICATION TABLE OF S}

\begin{tabular}{|c|l|l|l|l|}
\hline$\cdot$ & $a$ & $b$ & $c$ & $d$ \\
\hline$a$ & $a$ & $a$ & $a$ & $a$ \\
\hline$b$ & $a$ & $a$ & $d$ & $a$ \\
\hline$c$ & $a$ & $a$ & $a$ & $a$ \\
\hline$d$ & $a$ & $a$ & $a$ & $a$ \\
\hline
\end{tabular}

Further define I-V-FS $I$ on $S$ as:

$$
\tilde{f}_{I}(y)=\left\{\begin{array}{cc}
{[0,0],} & \text { if } y \in\{a, b\} \\
{[0.3,0.4],} & \text { if } y \in\{c, d\}
\end{array}\right.
$$

By routine calculation it can be shown that $I$ is $\mathrm{I}-\mathrm{V}$ $\left(\bar{\epsilon}_{00.40 .5]}, \bar{\epsilon}_{00.4,0.5]} \vee \bar{q}_{[0.5,0.6]}\right)$-FF of $S$.

Theorem 3.3 and Theorem 3.4 provided in the following lines are the main results of this paper. These results gives alternate definitions of I-V $\left(\bar{\epsilon}_{\tilde{\gamma}}, \bar{\epsilon}_{\tilde{\gamma}} \vee \bar{q}_{\tilde{\delta}}\right)$-FLF and I-V $\left(\bar{\epsilon}_{\bar{\gamma}}, \bar{\epsilon}_{\tilde{\gamma}} \vee \bar{q}_{\tilde{\delta}}\right)-F R F$. Further, these results are very helpful to prove all the other results of this paper.

\section{Theorem}

Let $I$ be an I-V-FS of $S$ and $x, y$ are arbitrary elements of $S$. Then $I$ is an I-V $\left(\bar{\epsilon}_{\tilde{\gamma}}, \bar{\epsilon}_{\tilde{\gamma}} \vee \bar{q}_{\tilde{\delta}}\right)$-FLF of $S$ if and only if the following statements are simultaneously true:

(1) $y \geq x$ implies $\widetilde{f}_{I}(x) \leq \operatorname{rmax}\left\{\widetilde{f}_{I}(y), \widetilde{\delta}\right\}$,

(2) $\mathrm{r} \min \left\{\tilde{f}_{I}(x), \tilde{f}_{I}(y)\right\} \leq \operatorname{rmax}\left\{\tilde{f}_{I}(x y), \tilde{\delta}\right\}$,

(3) $\tilde{f}_{I}(x y) \leq \mathrm{r} \max \left\{\tilde{f}_{I}(x), \widetilde{\delta}\right\}$.

Proof. Let $I$ be I-V $\left(\bar{\epsilon}_{\tilde{\gamma}}, \bar{\epsilon}_{\tilde{\gamma}} \vee \bar{q}_{\tilde{\delta}}\right)$-FLF and $a, b$ are in $S$ such that $a \leq b$ and $\operatorname{rmax}\left\{\widetilde{f}_{I}(b), \widetilde{\delta}\right\}<\tilde{f}_{I}(a)$. Then $\mathrm{r} \max \left\{\tilde{f}_{I}(b), \widetilde{\delta}\right\}<\widetilde{\theta} \leq \widetilde{f}_{I}(a)$ for some $\widetilde{\delta}<\widetilde{\theta} \leq[1,1]$ and this inequality implies $b_{\tilde{\theta}} \bar{\epsilon}_{\tilde{\gamma}} I$ but $a_{\tilde{\theta}} \in_{\tilde{\gamma}} I$ and $a_{\tilde{\theta}} q_{\tilde{\delta}} I$ (since $\left.\tilde{f}_{I}(a)+\widetilde{\theta}>\widetilde{\delta}+\widetilde{\delta}\right)$ a contradiction and hence (1) holds.

Consider (2) does not hold for some $a, b$ in $S$. Then we have $\quad \mathrm{r} \max \left\{\widetilde{f}_{I}(a b), \widetilde{\delta}\right\}<\widetilde{\theta}_{1} \leq \mathrm{r} \min \left\{\widetilde{f}_{I}(a), \widetilde{f}_{I}(b)\right\} \quad$ some $\widetilde{\delta}<\tilde{\theta}_{1} \leq[1,1]$. Clearly from the last inequality we see that $(a b)_{\tilde{\theta}_{1}} \bar{\epsilon}_{\tilde{\gamma}} I$ whereas $a_{\tilde{\theta}_{1}} \in \in_{\tilde{\gamma}} \wedge q_{\tilde{\delta}} I$ and $b_{\tilde{\theta}_{1}} \in_{\tilde{\gamma}} \wedge q_{\tilde{\delta}} I$. This is contradicting Statement $\left(d_{2}\right)$ of Definition 3.1. Hence (2) holds.
Next assume on contrary that (3) does not hold for $a, b$ in $S$, then $\operatorname{rmax}\left\{\tilde{f}_{I}(a), \widetilde{\delta}\right\}<\widetilde{\theta}_{2} \leq \tilde{f}_{I}(a b)$ for some $\tilde{\delta}<\widetilde{\theta}_{2} \leq[1,1]$. This inequality shows that $a_{\tilde{\theta}_{2}} \bar{\epsilon}_{\bar{\gamma}} A$ but $(a b)_{\widetilde{\theta}_{2}} \bar{\epsilon}_{\tilde{\gamma}} \wedge \bar{q}_{\widetilde{\delta}^{\prime}}$ a contradiction with Statement $\left(d_{3}\right)$ of Definition 3.1. Hence (3) also holds.

Conversely, let $I$ satisfy Statement (1), (2) and (3) simultaneously. If $x \leq y$ in $S$ and $y_{\tilde{\theta}} \bar{\epsilon}_{\tilde{\gamma}} I$, then $\widetilde{f}_{I}(y)<\widetilde{\theta}$ and by Statement (1) we have

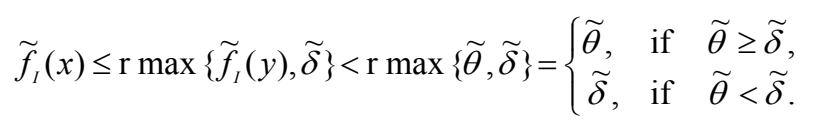

The above inequality shows that $x_{\tilde{\theta}} \bar{\epsilon}_{\tilde{\gamma}} \vee \bar{q}_{\tilde{\delta}} I$ (since $\widetilde{f}_{I}(x)<\widetilde{\theta}$ or $\left.\widetilde{f}_{I}(x)+\widetilde{\theta}<\widetilde{\delta}+\widetilde{\theta}<\widetilde{\delta}+\widetilde{\delta}\right)$. Hence Statement $\left(d_{1}\right)$ of Definition 3.1 holds for $y \geq x$.

Secondly, take $x, y$ from $S$ such that $(x y)_{\tilde{\theta}} \bar{\epsilon}_{\tilde{\gamma}} I$, then $\widetilde{f}_{I}(x y)<\widetilde{\theta}$ and by Statement (2) we have

$$
\begin{aligned}
\mathrm{r} \min \left\{\tilde{f}_{I}(x), \tilde{f}_{I}(y)\right\} \leq \mathrm{r} \max \left\{\tilde{f}_{I}(x y), \widetilde{\delta}\right\} & <\mathrm{r} \max \{\tilde{\theta}, \widetilde{\delta}\} \\
& = \begin{cases}\widetilde{\theta}, & \text { if } \quad \tilde{\theta} \geq \widetilde{\delta}, \\
\widetilde{\delta}, & \text { if } \quad \tilde{\theta}<\widetilde{\delta}\end{cases}
\end{aligned}
$$

From the above inequality we have $x_{\tilde{\theta}} \bar{\epsilon}_{\tilde{\gamma}} \vee \bar{q}_{\tilde{\delta}} I$ or $y_{\widetilde{\theta}} \bar{\epsilon}_{\tilde{\gamma}} \vee \bar{q}_{\widetilde{\delta}} I$. Hence Statement $\left(d_{2}\right)$ of Definition 3.1 holds for each $x, y$ in $S$.

Finally, if we take $x, y$ from $S$ such that $x_{\widetilde{\theta}} \bar{\epsilon}_{\tilde{\gamma}} I$, then by Statement (3)

$$
\widetilde{f}_{I}(x y) \leq \operatorname{rmax}\left\{\widetilde{f}_{I}(x), \widetilde{\delta}\right\}<\operatorname{rmax}\{\widetilde{\theta}, \widetilde{\delta}\}= \begin{cases}\widetilde{\theta}, & \text { if } \quad \tilde{\theta} \geq \widetilde{\delta} \\ \widetilde{\delta}, & \text { if } \quad \widetilde{\theta}<\widetilde{\delta}\end{cases}
$$

The last inequality implies that $(x y)_{\tilde{\theta}} \bar{\epsilon}_{\tilde{\gamma}} \vee \bar{q}_{\tilde{\delta}} I$. Thus Statement $\left(d_{3}\right)$ also holds for each $x, y$ in $S$.

\section{Theorem}

$I$ is an I-V $\left(\bar{\epsilon}_{\tilde{\gamma}}, \bar{\epsilon}_{\tilde{\gamma}} \vee \bar{q}_{\tilde{\delta}}\right)$-FRF of $S$ if and only if Statements (1) and (2) of Theorem 3.3 and Statement (4) given below hold simultaneously:

(4) $\mathrm{r} \max \left\{\tilde{f}_{I}(y), \tilde{\delta}\right\} \geq \tilde{f}_{I}(x y)$ for each $x, y$ in $S$.

Proof. To prove the direct part we suppose $I$ is an I-V $\left(\bar{\epsilon}_{\tilde{\gamma}}, \bar{\epsilon}_{\tilde{\gamma}} \vee \bar{q}_{\tilde{\delta}}\right)$-FRF and assume on contrary that Statement (4) does not hold for $a, b$ in $S$. Then $\mathrm{r} \max \left\{\tilde{f}_{I}(b), \widetilde{\delta}\right\}<\widetilde{f}_{I}(a b)$ and hence $\quad \mathrm{r} \max \left\{\tilde{f}_{I}(b), \tilde{\delta}\right\}<\widetilde{\theta} \leq \tilde{f}_{I}(a b)$ for some $\widetilde{\delta}<\tilde{\theta} \leq[1,1]$. The last inequality implies $b_{\tilde{\theta}} \bar{\epsilon}_{\tilde{\gamma}} I$ but 
$(a b)_{\tilde{\theta}} \bar{\epsilon}_{\tilde{\gamma}} \wedge \bar{q}_{\tilde{\delta}} I$ a contradiction with Statement $\left(d_{4}\right)$ and hence we have to accept that Statement (4) holds.

Conversely, take two arbitrary elements $x$ and $y$ from $S$ and consider $y_{\tilde{\theta}} \bar{\epsilon}_{\tilde{\gamma}} I$, then by Statement (4) the following inequality can be written:

$$
\widetilde{f}_{I}(x y) \leq \mathrm{r} \max \left\{\tilde{f}_{I}(y), \widetilde{\delta}\right\}<\operatorname{rmax}\{\widetilde{\theta}, \widetilde{\delta}\}=\left\{\begin{array}{lll}
\widetilde{\theta}, & \text { if } & \widetilde{\theta} \geq \widetilde{\delta} \\
\widetilde{\delta}, & \text { if } & \widetilde{\theta}<\widetilde{\delta} .
\end{array}\right.
$$

The last inequality implies that $(x y)_{\tilde{\theta}} \bar{\epsilon}_{\tilde{\gamma}} \vee \bar{q}_{\tilde{\delta}} I$ and thus $\left(d_{4}\right)$ holds. The remaining part of the proof can be seen from Theorem 3.3.

From Theorems we can say that $I$ is I-V $\left(\bar{\epsilon}_{\tilde{\gamma}}, \bar{\epsilon}_{\bar{\gamma}} \vee \bar{q}_{\tilde{\delta}}\right)$-FF of $S$ if and only if Statements (1), (2), (3) of Theorem 3.3 and Statement (4) of Theorem 3.4 hold simultaneously for each element of $S$.

The following two corollaries arises by putting respectively $\widetilde{\gamma}=[0,0]$ and $\widetilde{\delta}=[0.5,0.5]$ in Theorem 3.3 and Theorem 3.4.

\section{E. Corollary}

Let $x, y$ be arbitrary members of $S$ and $I$ be I-V-FS of $S$. Then the following Statements are equivalent:

(5) The following inequalities hold:

(5.1) $y \geq x$ implies $\tilde{f}_{I}(x) \leq \operatorname{rmax}\left\{\tilde{f}_{I}(y),[0.5,0.5]\right\}$,

(5.2) $\mathrm{r} \min \left\{\tilde{f}_{I}(x), \tilde{f}_{I}(y)\right\} \leq \mathrm{r} \max \left\{\tilde{f}_{I}(x y),[0.5,0.5]\right\}$,

(5.3) $\tilde{f}_{I}(x y) \leq \mathrm{r} \max \left\{\tilde{f}_{I}(x),[0.5,0.5]\right\}$.

(6) $I$ is I-V $(\bar{\epsilon}, \bar{\in} \vee \bar{q})-\mathrm{FLF}$ of $S$.

\section{F. Corollary}

$I$ is I-V $(\bar{\epsilon}, \bar{\in} \vee \bar{q})$-FRF of $S$ if and only if Statements (5.1), (5.2) of Corollary 3.5 and the following statement hold simultaneously for each $x, y$ in $S$ :

(7) $\tilde{f}_{I}(x y) \leq \operatorname{rmax}\left\{\tilde{f}_{I}(y),[0.5,0.5]\right\}$.

The following two results (Theorem 3.7 and Theorem 3.8) link left (resp. right) filters with I-V $\left(\bar{\epsilon}_{\bar{\gamma}}, \bar{\epsilon}_{\bar{\gamma}} \vee \bar{q}_{\tilde{\delta}}\right)$-FLF (resp. I$\left.\mathrm{V}\left(\bar{\epsilon}_{\tilde{\gamma}}, \bar{\epsilon}_{\tilde{\gamma}} \vee \bar{q}_{\tilde{\delta}}\right)-\mathrm{FRF}\right)$ by means of level subset $(U(I ; \tilde{\theta}))$.

\section{G. Theorem}

Consider the level subset $U(I ; \widetilde{\theta})$ of $I$ be non-empty for each $\widetilde{\delta}<\widetilde{\theta} \leq[1,1]$. Then $U(I ; \widetilde{\theta})$ is a left filter of $S$ if and only if $I$ is $\mathrm{I}-\mathrm{V}\left(\bar{\epsilon}_{\tilde{\gamma}}, \bar{\epsilon}_{\tilde{\gamma}} \vee \bar{q}_{\tilde{\delta}}\right)$-FLF of $S$.

Proof. First we suppose $U(I ; \tilde{\theta})$ be a left filter. Let $x, y$ be arbitrary elements in $S$ with $y \geq x$ such that
$\tilde{f}_{I}(x)>\operatorname{rmax}\left\{\tilde{f}_{I}(y), \widetilde{\delta}\right\}$ that is Statement (1) of Theorem 3.3 does not hold. Then for some $\tilde{\delta}<\tilde{\theta} \leq[1,1]$ we have $\mathrm{r} \max \left\{\tilde{f}_{I}(y), \tilde{\delta}\right\}<\tilde{\theta} \leq \tilde{f}_{I}(x)$. This inequality implies that $y \notin U(I ; \widetilde{\theta})$ while $x \in U(I ; \widetilde{\theta})$, a contradiction with $\left(b_{1}\right)$. Hence Statement (1) of Theorem 3.3 holds.

Consider Statement (2) of Theorem 3.3 does not hold for arbitrary elements $x, y$ in $S$ that is $\mathrm{r} \min \left\{\tilde{f}_{I}(x), \tilde{f}_{I}(y)\right\}>\mathrm{r} \max \left\{\tilde{f}_{I}(x y), \tilde{\delta}\right\}$. Then for some $\tilde{\delta}<\tilde{\theta} \leq[1,1] \quad$ we have the inequality $\mathrm{r} \min \left\{\tilde{f}_{I}(x), \tilde{f}_{I}(y)\right\} \geq \widetilde{\theta}>\mathrm{r} \max \left\{\tilde{f}_{I}(x y), \widetilde{\delta}\right\} \quad$. The last inequality impels $x y \notin U(I ; \widetilde{\theta})$ while $x, y \in U(I ; \widetilde{\theta})$ and this contradicts $\left(b_{2}\right)$. Therefore, we must accept that Statement (2) of Theorem 3.3 holds.

Next we consider Statement (3) of Theorem 3.3 does not hold for some $x, y$ in $S$ that is $\tilde{f}_{I}(x y)>\operatorname{rmax}\left\{\tilde{f}_{I}(x), \widetilde{\delta}\right\}$. Then we can find some $\tilde{\delta}<\tilde{\theta} \leq[1,1]$ such that $\mathrm{r} \max \left\{\widetilde{f}_{I}(x), \widetilde{\delta}\right\}<\widetilde{\theta} \leq \widetilde{f}_{I}(x y)$. From this inequality clearly we can see that $x \notin U(I ; \widetilde{\theta})$ whereas $x y \in U(I ; \widetilde{\theta})$, a contradiction with $\left(b_{3}\right)$ and hence $\mathrm{r} \max \left\{\widetilde{f}_{I}(x), \widetilde{\delta}\right\} \geq \widetilde{f}_{I}(x y)$ for all $x, y \in S$. In the light of Theorem 3.3, the above discussion conclude that $I$ is I-V $\left(\bar{\epsilon}_{\bar{\gamma}}, \bar{\epsilon}_{\bar{\gamma}} \vee \bar{q}_{\tilde{\delta}}\right)$-FLF of $S$.

Conversely; let $I$ be $\mathrm{I}-\mathrm{V} \quad\left(\bar{\epsilon}_{\bar{\gamma}}, \bar{\epsilon}_{\bar{\gamma}} \vee \bar{q}_{\tilde{\delta}}\right)$-FLF and $U(I ; \widetilde{\theta}) \neq \phi$ for all $\widetilde{\delta}<\widetilde{\theta} \leq[1,1]$. Let $x, y$ are arbitrary elements in $S$ such that $x \leq y$ and $x \in U(I ; \tilde{\theta})$. Then by Statement (1) of Theorem 3.3 we have $\mathrm{r} \max \left\{\widetilde{f}_{I}(y), \widetilde{\delta}\right\} \geq \widetilde{f}_{I}(x) \geq \widetilde{\theta} \quad$. This inequality implies $y \in U(I ; \widetilde{\theta})$ and hence $\left(b_{1}\right)$ holds.

Secondly, if $x \in U(I ; \widetilde{\theta})$ and $y \in U(I ; \widetilde{\theta})$, then by Statement (2) of Theorem 3.3 we have the following inequality:

$$
\mathrm{r} \max \left\{\tilde{f}_{I}(x y), \widetilde{\delta}\right\} \geq \mathrm{r} \min \left\{\tilde{f}_{I}(x), \tilde{f}_{I}(y)\right\} \geq \mathrm{r} \min \{\widetilde{\theta}, \widetilde{\theta}\}=\widetilde{\theta},
$$

follows that $x y \in U(I ; \tilde{\theta})$ and hence $\left(b_{2}\right)$ also holds.

Finally, we assume that $x y \in U(A ; \tilde{t})$ then by Statement (3) Theorem 3.3 we have $\mathrm{r} \max \left\{\tilde{f}_{I}(x), \widetilde{\delta}\right\} \geq \widetilde{f}_{I}(x y) \geq \widetilde{\theta}$. This inequality shows that $x \in U(I ; \widetilde{\theta})$ and hence $\left(b_{3}\right)$ also holds. This completes the proof.

\section{H. Theorem}

Let $U(I ; \widetilde{\theta})$ be non-empty for every $\widetilde{\delta}<\widetilde{\theta} \leq[1,1]$. Then $U(I ; \tilde{\theta})$ is right filter of $S$ if and only if $I$ is I-V $\left(\bar{\epsilon}_{\bar{\gamma}}, \bar{\epsilon}_{\tilde{\gamma}} \vee \bar{q}_{\tilde{\delta}}\right)$ -FRF of $S$.

Proof. The proof easy and hence omitted. 
Cleary, I-V-FLF implies I-V $\left(\bar{\epsilon}_{\tilde{\gamma}}, \bar{\epsilon}_{\tilde{\gamma}} \vee \bar{q}_{\tilde{\delta}}\right)$-FLF but conversely it is not true. The following example is constructed I support of this claim.

\section{Example}

From Example 3.3 we observe that the $I$ is $\mathrm{I}-\mathrm{V}$ $\left(\bar{\epsilon}_{0.4,0.5]}, \bar{\epsilon}_{0.4,0.5]} \vee \bar{q}_{[0.5,0.6]}\right)$-FLF but not an I-V-FLF, since $\widetilde{f}_{I}(b)=[0,0]<\widetilde{f}_{I}(b c)=\widetilde{f}_{I}(d)=[0.3,0.4]$.

Now it is natural to ask that under which circumstances an I$\mathrm{V}\left(\bar{\epsilon}_{\tilde{\gamma}}, \bar{\epsilon}_{\tilde{\gamma}} \vee \bar{q}_{\tilde{\delta}}\right)-$ FLF will be I-V-FLF. In this regards, we provide the following theorem answers to this question.

\section{J. Theorem}

Let $I$ be $\mathrm{I}-\mathrm{V}\left(\bar{\epsilon}_{\tilde{\gamma}}, \bar{\epsilon}_{\tilde{\gamma}} \vee \bar{q}_{\tilde{\delta}}\right)-\mathrm{FLF}$ such that $\tilde{f}_{I}(y) \geq \widetilde{\delta}$ for every $y$ in $S$. Then $I$ is I-V-FLF.

Proof. Choose arbitrary elements $x, y$ from $S$ such that $x \leq y$ and assume that $I$ is $\mathrm{I}-\mathrm{V}\left(\bar{\epsilon}_{\tilde{\gamma}}, \bar{\epsilon}_{\tilde{\gamma}} \vee \bar{q}_{\tilde{\delta}}\right)$-FLF. Then by Statement (1) of Theorem 3.3 we have the inequality $\mathrm{r} \max \left\{\tilde{f}_{I}(y), \widetilde{\delta}\right\} \geq \widetilde{f}_{I}(x)$ but by hypothesis $\tilde{f}_{I}(y)$ cannot be less than $\widetilde{\delta}$. Therefore, $\mathrm{r} \max \left\{\tilde{f}_{I}(y), \widetilde{\delta}\right\}=\widetilde{f}_{I}(y)$ and from the last inequality we have $\tilde{f}_{I}(y) \geq \widetilde{f}_{I}(x)$. Hence $\left(b_{5}\right)$ holds.

Next, we choose any arbitrary elements $x, y$ from $S$. Then Statement (2) of Theorem 3.3 implies that $\mathrm{r} \max \left\{\tilde{f}_{I}(x y), \widetilde{\delta}\right\} \geq \mathrm{r} \min \left\{\tilde{f}_{I}(x), \tilde{f}_{I}(y)\right\} \quad$ but by hypothesis $\widetilde{f}_{I}(x y)$ cannot be less than $\tilde{\delta}$. Therefore, we have $\tilde{f}_{I}(x y) \geq \mathrm{r} \min \left\{\tilde{f}_{I}(x), \tilde{f}_{I}(y)\right\}$ and hence $\left(b_{6}\right)$ also holds.

Finally, from Statement (3) of Theorem 3.3 we have $\mathrm{r} \max \left\{\widetilde{f}_{I}(x), \widetilde{\delta}\right\} \geq \widetilde{f}_{I}(x y)$ but by hypothesis $\widetilde{f}_{I}(x)$ cannot be less than $\widetilde{\delta}$. Therefore, $\mathrm{r} \max \left\{\tilde{f}_{I}(x), \widetilde{\delta}\right\}=\widetilde{f}_{I}(x)$ and hence we have $\tilde{f}_{I}(x) \geq \tilde{f}_{I}(x y)$. Therefore, $\left(b_{7}\right)$ also holds. The above discussion concludes in the light of Theorem 3.3 that $I$ is I-VFLF of $S$. Hence, we say that I-V $\left(\bar{\epsilon}_{\tilde{\gamma}}, \bar{\epsilon}_{\tilde{\gamma}} \vee \bar{q}_{\tilde{\delta}}\right)$-FLF implies IV-FLF whenever $\tilde{f}_{I}(y) \geq \widetilde{\delta}$ for every $y$ in $S$.

\section{K. Theorem}

If $\tilde{f}_{I}(x) \geq \widetilde{\delta}$ for every $x$ in $S$, then I-V $\left(\bar{\epsilon}_{\tilde{\gamma}}, \bar{\epsilon}_{\tilde{\gamma}} \vee \bar{q}_{\tilde{\delta}}\right)$-FRF implies I-V-FRF.

Proof. The proof is easy and hence omitted.

\section{CONCLUSION}

Ordered semigroups play a key role in the field of mathematics with a large number of applications in different areas like coding theory, automata theory and sequential machine, language theory and formal grammar and neural networks. We employed the existing concept of I-V-FS ordered semigroups and added another generalization of fuzzy filters to the literature. This new generalized form of fuzzy filters is called $\operatorname{I-V}\left(\bar{\epsilon}_{\tilde{\gamma}}, \bar{\epsilon}_{\tilde{\gamma}} \vee \bar{q}_{\tilde{\delta}}\right)$-FFs. In this study we investigated a condition under which I-V $\left(\bar{\epsilon}_{\tilde{\gamma}}, \bar{\epsilon}_{\tilde{\gamma}} \vee \bar{q}_{\tilde{\delta}}\right)$-FFs are ordinary I-V-FFs. In addition, ordinary I-V-FFs is linked with this new concept.

\section{ACKNOWLEDGMENT}

This work was supported by the National Natural Science Foundation of China (11571378) and University of Malakand.

\section{REFERENCES}

[1] M. B. Gorzałczany, A method of inference in approximate reasoning based on interval-valued fuzzy sets, Fuzzy sets and Systems, Vol 21, No. 1, pp. 1-17, 1987.

[2] L. A. Zadeh, The concept of a linguistic variable and its application to approximate reasoning-I. Information sciences, Vol 8, pp. 199-249, 1975.

[3] I. Grattan - Guinness, Fuzzy Membership Mapped onto Intervals and Many - Valued Quantities, Mathematical Logic Quarterly, Vol 22, pp. 149-160, 1976.

[4] K. U. Jahn, Intervall - wertige Mengen, Mathematische Nachrichten, Vol 68, pp. 115-132, 1975.

[5] A. Rosenfeld, Fuzzy groups, Journal of Mathematical Analysis and Applications, Vol 35, No. 3, pp. 512-517, 1971.

[6] R. Biswas,. Rosenfeld's fuzzy subgroups with interval-valued membership functions, Fuzzy sets and Systems, Vol 63, No. 1, pp. 87-90, 1994.

[7] B. Davvaz, Interval-valued fuzzy subhypergroups, Korean Journal of Computational and Applied Mathematics, Vol 6, pp. 197-202, 1999.

[8] A. Narayanan, and T. Manikantan, Interval-valued fuzzy ideals generated by an interval-valued fuzzy subset in semigroups, Journal of Applied Mathematics and Computing, Vol 20, pp. 455-464, 2006.

[9] M. Shabir and I. A. Khan, Interval-valued fuzzy ideals generated by an interval-valued fuzzy subset in ordered semigroups, Mathware \& Soft Computing, Vol 15, pp. 263-272, 2008.

[10] P. Pao-Ming and L. Ying-Ming, Fuzzy topology I Neighborhood structure of a fuzzy point and Moore-Smith convergence, Journal of Mathematical Analysis and Applications, Vol 76, pp. 571-599, 1980.

[11] V. Murali, Fuzzy points of equivalent fuzzy subsets, Information sciences, Vol 158, pp. 277-288, 2004.

[12] S. Bhakat and P. Das, $(\in, \in \vee q)$-fuzzy subgroup, Fuzzy sets and Systems, Vol 80, No. 3, pp. 359-368, 1996.

[13] B. Davvaz and A. Khan, Generalized fuzzy filters in ordered semigroups, Iranian Journal of Science \& Technology A 1, pp. 77-86, 2012.

[14] N. Kehayopulu and M. Tsingelis, Fuzzy sets in ordered groupiods, Semigroup Forum, Vol 65, pp. 128-132, 2002.

[15] Y. B. Jun, B. Davvaz, and A. Khan, Filters of ordered semigroups based on the fuzzy points, International Journal of Fuzzy Systems, Vol 24, No. 3, pp. 619-630 2013.

[16] B. Davvaz, A. Khan, N. H. Sarmin and H. Khan, More General Forms of Interval Valued Fuzzy Filters of Ordered Semigroups, International Journal of Fuzzy Systems, Vol 15, No. 2, pp. 110-126, 2013.

[17] H.U. Khan, F. M. Khan, A. Khan and N. H. Sarmin, New generalisation of interval-valued fuzzy filters of ordered semigroups, Sindh Univ. Res. Jour. (Sci. Ser.) Vol 48, No. 2, pp. 383-388, 2016. 